\title{
Colour perception in pathologists: the Farnsworth-Munsell 100-hue test
}

\author{
H S Rigby, B F Warren, J Diamond, C Carter, J W B Bradfield
}

\begin{abstract}
The value of many histological stains depends on the ability of the observer to differentiate colour. This ability was assessed in 30 histopathologists and cytopathologists of varying experience using the Farnsworth-Munsell 100-hue test. As a group, the pathologists performed better than a reference population. Twenty eight subjects showed a wide ranging ability to differentiate colour: none was colour blind. Three of the 30 pathologists, however, fell below the twentieth centile for normal subjects and only one was aware of this deficiency! They may unknowingly misinterpret subtle stains. Two of these three had specific and major defects which could affect their ability to interpret a wide range of less subtle stains. Those with the poorest colour discrimination were not those with the least experience of microscopy.

Pathologists should be apprised of the importance of their ability to discriminate colour, and that formal colour vision testing of prospective histopathologists may be appropriate.
\end{abstract}

The interpretation of many tinctorial stains depends on the ability of the histopathologist to distinguish hues. It has been assumed, without formal testing, that histopathologists are competent colour discriminators. The reason for this study was the inability of one of the authors to distinguish the differential staining of colonic mucin with the KOH AB1/ $\mathrm{mPas}$ technique. ${ }^{1}$ Correct interpretation of this particular dual staining method requires the ability to distinguish blue/purple from red/purple to differentiate sulphomucin from sialomucin. ${ }^{2}$ A standard test of colour vision, the Ishihara test, failed to show any difference between the two subjects. Therefore, a more specific test of colour discrimination-the Farnsworth-Munsell 100-hue test was used. ${ }^{3}$ This showed that the former observer had inferior colour discrimination, in spite of better visual acuity.

\section{Methods}

The Farnsworth-Munsell 100-hue test is a simple method for measuring colour discrimination. In industry it is used to test employees in colour control laboratories involved in the manufacture of paints and dyes. $^{3}$

The test requires each subject to sort into a colour graded order a series of randomly arranged coloured caps, from the whole spectrum, into each of four black metal boxes (figs 1 and 2). Each box contained discs relevant to part of the colour spectrum, plus two fixed reference coloured discs, one at each end. During the test each subject was seated at a table at a North facing window between 11.00 and 12.00 hours, in an attempt to achieve the prescribed lighting conditions of Farnsworth and Munsell, ${ }^{3}$ who proposed either a North facing window on a slightly overcast day, or a Maclaren lamp set to deliver $6740^{\circ}$ Kelvin at 25 foot-candles, or a $6500^{\circ}$ daylight fluorescent tube. The tests were all carried out in September.

At the end of the test the four boxes were closed and inverted individually by the examiner. The underside of each coloured cap carried a sequential number from 1 to 85 .

The test score for each subject was calculated as follows. The number of each cap was compared with those on the immediately adjacent caps. The differences between the number on the test cap and each of its neighbours were added together. A perfect score for each cap would therefore have been 2-for example, in the case of cap 4 the calculation was as follows: $4-3=1,5-4=1$, and the sum of these differences is 2 . The error score for an individual cap is given by the differences between the abnormal score and the expected perfect score of 2.

For each subject tested the error scores were plotted on a Farnsworth-Munsell chart (fig 3). This chart comprises a central disc which representes the entire colour spectrum. Outside this are concentric rings at error score intervals of 1 . The innermost circle represents a perfect score of 2 .

Twenty eight volunteer histopathologists and two cytopathologists ( 23 men and seven women) were studied. These pathologists were of varying experience and included 10 consultants and two experienced non-medical cytologists, 11 senior trainees, and seven junior trainees. Eleven of the subjects were aged 20-34, 12 were aged 35-44, and seven were aged 45 or more years.

The total number of errors made by each pathologist was calculated and compared with those of a standard reference population of prospective employees in the paint and dye industry (Farnsworth). Any population can be 


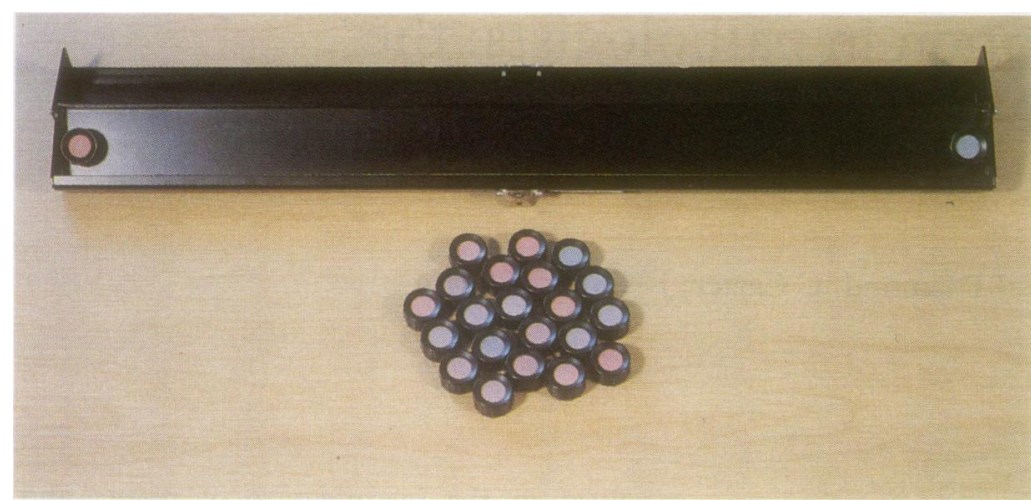

Figure 1 Randomly arranged coloured caps of one box.

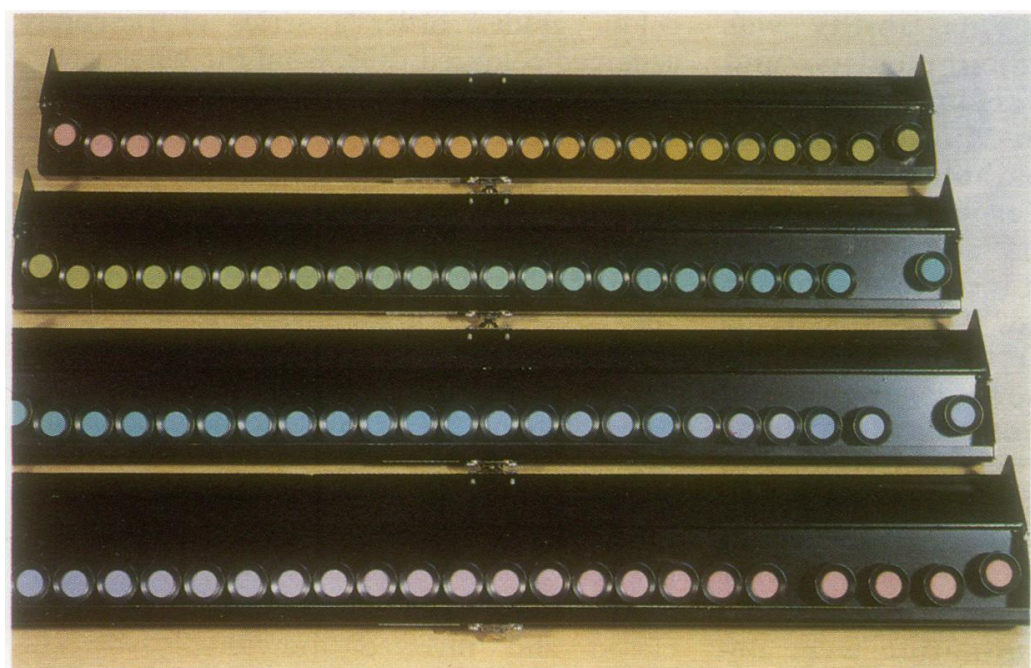

Figure 2 Perfect arrangement of coloured caps in the four boxes. divided into those of superior ability (less than 17 errors), average ability (20-100 errors), and low ability (more than 100 errors), based on the total number of error scores. In the standard reference population $16 \%$ were of low ability, $68 \%$ were of average ability, and $16 \%$ were of superior ability. ${ }^{3}$

The error scores in each of the groups were analysed using the Kruskal-Wallis one way analysis of variance.

\section{Results}

Examples of error charts are shown in figs 3 to 8 . Taken together, nine out of $30(30 \%)$ pathologists had superior competence for colour discrimination compared with $16 \%$ of the reference population and three out of $30(10 \%)$ had inferior competence for colour discrimination compared with $16 \%$ of the reference population. Eighteen ( $40 \%$ ) of the pathologists were of average competence for colour discrimination compared with $68 \%$ of the reference population.

There was no statistical difference in the error scores among the three groups- consultants and non-medical cytopathologists, senior trainees, and junior trainees (table 1). Similarly no difference was seen in the error scores among different age groups (table 2), or for either sex (table 3).
Table 1 Results of error scores according to employee status

\begin{tabular}{lrcl}
$\begin{array}{l}\text { Consultants and non- } \\
\text { medical cytologists }\end{array}$ & Senior trainees & Junior trainees \\
\hline 1 & 8 & 29 & 32 \\
2 & 50 & 43 & 56 \\
3 & 26 & 46 & 62 \\
4 & 14 & 14 & 24 \\
5 & 24 & 12 & 95 \\
6 & 4 & 45 & 16 \\
7 & 84 & 108 & 36 \\
8 & 62 & 108 & \\
9 & 16 & 16 & \\
10 & 69 & 69 & \\
11 & 80 & 514 & \\
12 & 4 & & \\
\hline$(p=0 \cdot 4443)$ & &
\end{tabular}

Table 2 Results of error scores according to age

\begin{tabular}{rrlr}
\hline & $20-34$ & $35-44$ & $45+$ \\
\hline 1 & 32 & 45 & 50 \\
2 & 36 & 46 & 14 \\
3 & 16 & 14 & 108 \\
4 & 8 & 26 & 69 \\
5 & 29 & 12 & 4 \\
6 & 43 & 24 & 62 \\
7 & 514 & 95 & 84 \\
8 & 108 & 24 & \\
9 & 16 & 16 & \\
10 & 62 & 69 & \\
11 & 4 & 56 & \\
12 & & 80 & \\
\hline
\end{tabular}

$(p=0.8838)$

Table 3 Results of error scores according to sex

\begin{tabular}{rrr}
\hline & Women & Men \\
\hline 1 & 32 & 16 \\
2 & 36 & 4 \\
3 & 50 & 24 \\
4 & 4 & 69 \\
5 & 43 & 24 \\
6 & 8 & 26 \\
7 & 29 & 16 \\
8 & & 108 \\
9 & & 514 \\
10 & 45 \\
11 & & 12 \\
12 & & 14 \\
13 & & 62 \\
14 & & 56 \\
15 & & 95 \\
16 & & 69 \\
17 & & 62 \\
18 & & 84 \\
19 & & 14 \\
20 & & 16 \\
21 & & 80 \\
22 & & 46 \\
23 & &
\end{tabular}

\section{Discussion}

There are three facets in the histopathologist's diagnostic armamentarium. These are pattern recognition, ${ }^{4}$ the ability to discriminate colour, and knowledge. These abilities are never. considered when appointing new trainees in histopathology. Moreover, a whole career may pass without any formal assessment of colour discrimination. The development of more subtle tinctorial stains takes no account of the inability of the requesting histopathologist to distinguish their hues.

We are unaware of any previous study of colour discrimination in histopathologists. This study has shown that as a population pathologists in Bristol performed better than a standard reference population, but three pathologists were previously unaware of their 
Figure 3 FarnsworthMunsell chart showing a perfect score marked at concentric ring 2.

Figure 4 Chart of pathologist of superior ability.

Figure 5 Chart of pathologist of average ability.

Figure 6 Chart of pathologist of low ability.

Figure 7 Chart of a pathologist with a previously known red green colour blindness.

Figure 8 Chart of a pathologist with a previously unknown major defect in colour discrimination.

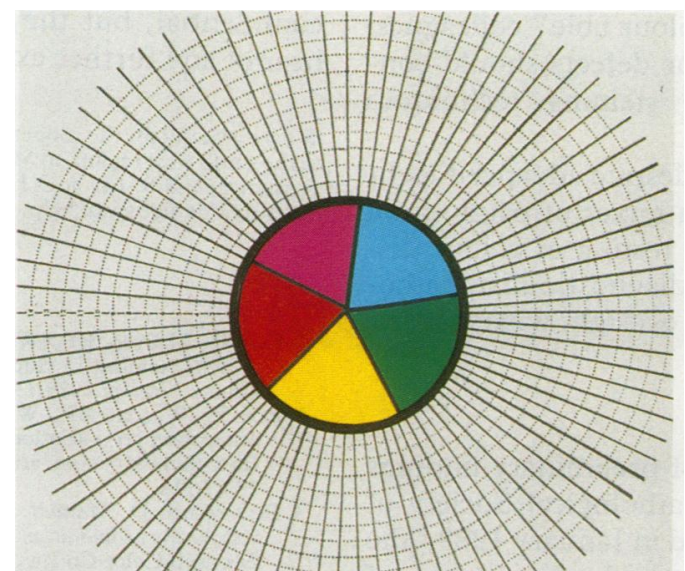

3

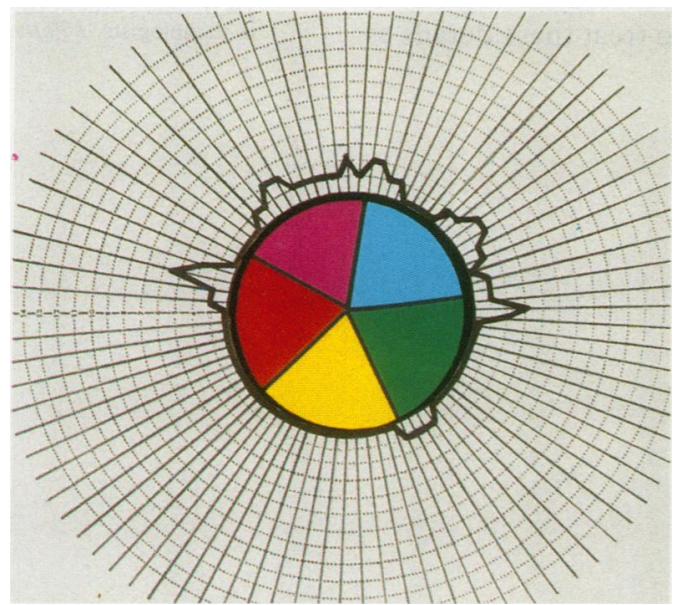

5

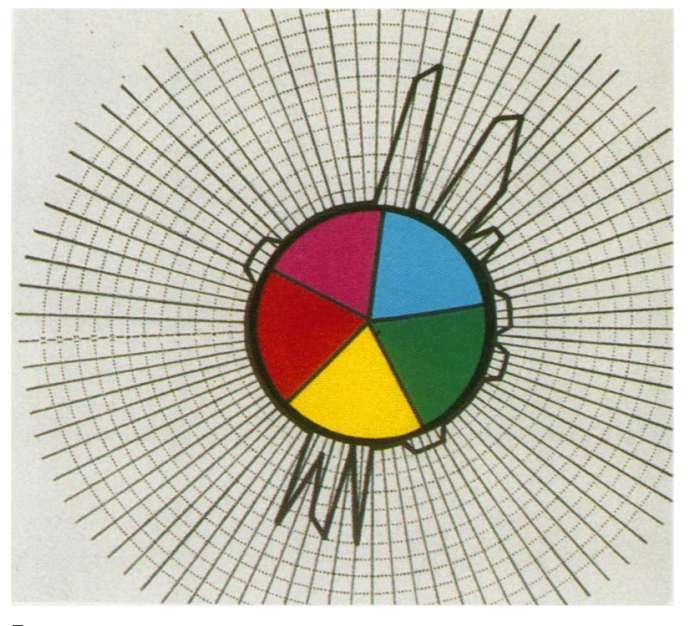

low ability to discriminate hues. None of the people in the low ability group was a woman. The ability of pathologists to discriminate colours was not related to age or professional experience.

Two of the pathologists tested had specific and major defects. One of these defects was previously known. The other was only recognised for the first time as a result of this study. The implication is that among a group of people in whom the ability to discriminate colours is a vital part of their professional life there will be individuals with an unrecognised

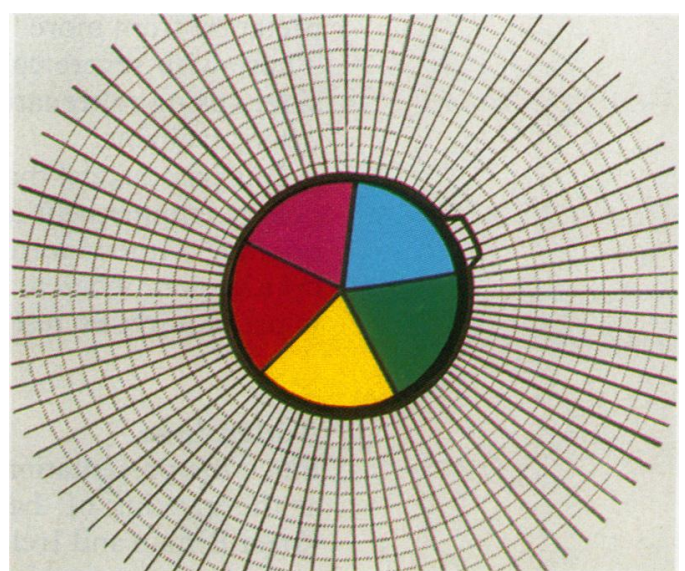

4
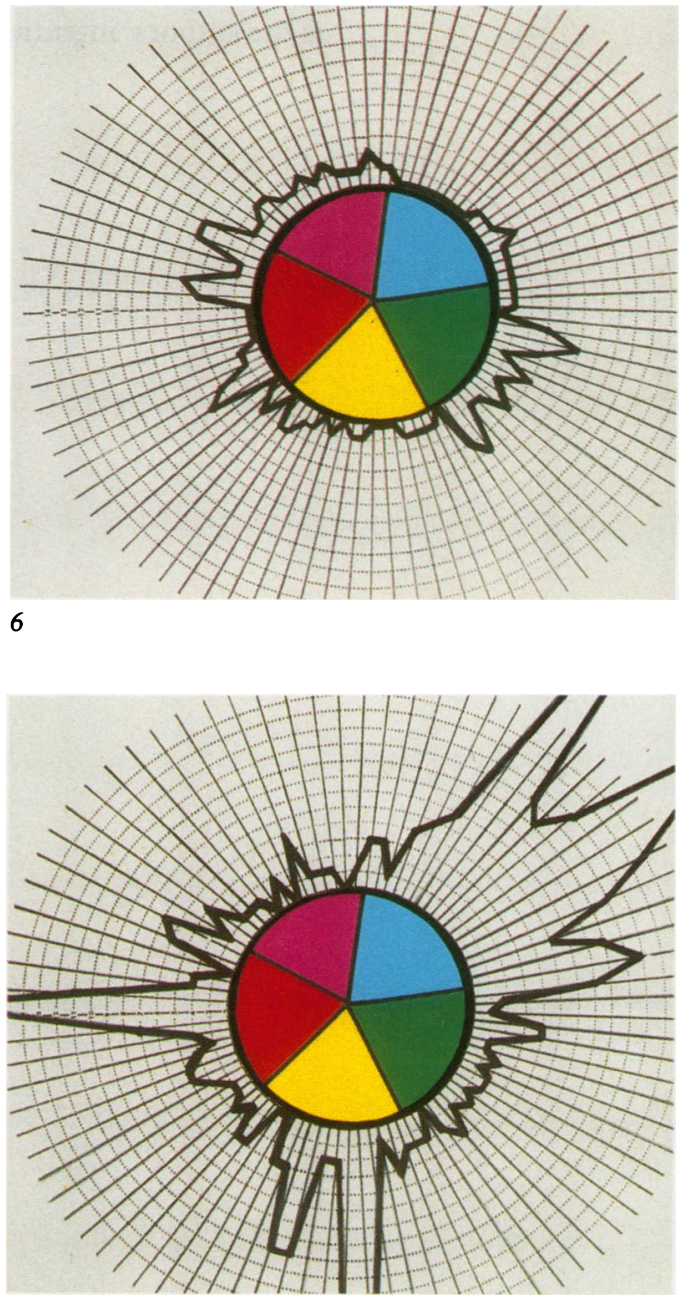

inability to do so. Pathologists with known bipolar colour blindness have frequently adapted their staining techniques to become more reliant on shade. Our evidence suggests that difficulties will be encountered when interpreting certain stains if the operator's ability to distinguish colours falls below the twentieth centile for normal. We therefore recommend that pathologists using these new techniques ${ }^{1}$ should formally assess their colour vision using the Farnsworth-Munsell 100-hue test. Those with below average ability to discriminate colour could then seek confirmation of inter- 
pretation from more "colour able" colleagues. Those with severe colour defects should consider using alternative staining techniques where available.

It is paradoxical that despite advanced technology employment as a mixer of paint is not possible without formal testing of colour discrimination, whereas a diagnostic histopathologist requires no test of eyesight whatsoever.

\section{Addendum}

Since the presentation of preliminary findings at the meeting of the Pathological Society of Great Britain and Ireland in January $1989,{ }^{5}$ the authors have received anecdotal evidence of varying degrees of colour blindness in several eminent past and present histopathologists. It is the authors' intention to treat these details as confidential, but they would be interested to hear of any further examples.

We are grateful for the cooperation of the volunteer pathologists in the south west region, to $\mathrm{Mr} \mathrm{C} \mathrm{C}$ Jeal for expert photographic assistance, and to $\mathrm{Mr} \mathrm{A} \mathrm{O}$ Hughes, senior lecturer in medical statistics, for statistical help.

1 Roe R, Warren BF, Williamson RCN, Bradfield JWB. Mucin staining in transitional mucosa in right and left colon. J Pathol 1989;157:174A

2 Roe R, Warren BF, Williamson RCN, Bradfield JWB Interobserver variation in colour assessment in mucin histochemistry-an attempt at control. J Pathol 1989 157:169A.

3 Farnsworth D Manual: The Farnsworth Munsell 100 Hue test for the examination of colour discrimination. Baltimore: test for the examination of colour

4 Berbaum KS, Platz C. Pattern recognition. Hum Pathol 1988;19:1127-31.

5 Rigby HS, Warren BF, Diamond J, Carter C, Bradfield JWB. The Farnsworth Munsell test of colour perception in pathologists. J Pathol 1989;158:343A. 